\title{
Combining Ionic Computer Models to Follow the Propagation of Electrical Activity of Atrial Fibrillation in the Left Atrium
}

\author{
Saaima Ahmad 1, Mahmoud Ehnesh 1, P J Stafford 2, 3, \\ G André $\mathrm{Ng}$ 2, 3, Fernando S Schlindwein 1, 2, 3 \\ 1 School of Engineering, University of Leicester \\ 2 Department of Cardiovascular Sciences, University of Leicester \\ 3 NIHR Leicester Cardiovascular Biomedical Research Centre
}

\begin{abstract}
To better understand the propagation of electrical activity of Atrial Fibrillation (AF) in the Left Atrium (LA), is essential for AF termination and treatment (catheter ablation). Components of 3 computing models were combined, they were: Fenton \& Karma, Courtemanche et al. and Simitev-Biktashev. It aims to follow the spread of $A F$ in simulation form in the LA and be generalisable, using a larger set of parameters, achieved by combining models. Potentially, enabling a degree of efficiency in trailing the propagation of $A F$ in the $L A$ in an individualised manner for each patient. Retrospective data from 10 subjects who underwent persistent $A F$ (PersAF) ablation for the first time was placed over stimulation protocol of the amalgamated model on MATLAB.3D simulations on subject data were run creating virtual visualisation of the propagation and entropy of $A F$ in the LA. Results were verified by determining singularly high dominant frequency (HDF) and phase analysis. Using code combining the 3 models on MATLAB retrieved $3 D$ simulations for all 10 patients, with a success rate of propagation trailing of $79 \pm 5 \%$. Propagation route coincided with HDF regions in $66 \%$ of patient data. Future research should involve developing the code into a standard subset to follow propagation of $A F$ in real time during ablation procedures.
\end{abstract}

\section{Introduction}

The prominence of $\mathrm{AF}$ is on the incline in the $\mathrm{UK}$, with roughly 1.3 million diagnosed [3,4]. Therefore, the need for research to investigate methodology to streamline current treatment methods is paramount. Currently, catheter ablations are most commonly used in clinical practice as a non-pharmacological approach [4]. This method uses 3D electroanatomic geometry created from Atrial Electrograms collected via electrophysiology studies. This approach is prone to subjectivity, specifically in determining where an ablation point should be made. Previous research using frequency analysis of dominant frequency (DF) patterns has shown evidence of re-entry circuits and phase patterns $[12,17,18]$. Alongside these measures, computer model simulations can further track the propagation of electrical activity in the LA during AF increasing the overall objectivity during ablation procedures. Components of 3 computing models are combined, they are: Fenton \& Karma, Courtmanche et al. and Simitev-Biktashev [6,9,13]. This paper aims to investigate whether such a combination can follow the spread of AF in simulation form in the LA and be generalisable, using a larger set of parameters. Potentially, improving tracking the propagation of $\mathrm{AF}$ in the LA in an individualised manner for each patient. The second aim of the paper is investigating if the results from the latter stage agree with phase analysis and DF maps.

\section{Methodology}

\subsection{Subject Recruitment}

The retrospective data used to test the computer model was via the following subject recruitment. This investigation enlisted 10 PersAF patients from Glenfield Hospital (University Hospital of Leicester), all of whom had not undergone catheter ablation (with a 2048 node detection catheter) in the LA beforehand. The ages of the recruited patients ranged from 36 to 76 . Ethical requirements and formal ethical approval were obtained from the local trust ethical committee. For each patient, individual informed consent was sought before each procedure.

\subsection{Atrial Electrograms Data Acquisition}

The instrument used to collect AEG data during the ablation procedure is a high-density non-contact mapping multi-electrode array catheter. The data collected from the mapping catheter via the commercial system forms 3D LA geometry maps in real-time. Specific anatomic points 
are labelled for objectivity, these are left atrial appendage, atrial roof, anterior wall, posterior wall, pulmonary veins, mitral valve and septum. The data collected were bipolar AEG recordings at baseline and post procedure, with a band pass filter of $30-250 \mathrm{~Hz}$ and notch filtered at $50 \mathrm{~Hz}$ the AEGs were taken for 5-minute segments. The use of clinical retrospective data is facilitated by the license the University of Leicester holds with Ensite at Glenfield Hospital.

\subsection{Signal Processing}

Data collected from the virtual electrograms via the catheters is exported in 5-minute segments from the commercial system into MATLAB (R2018a: MathWorks). To this data QRST subtraction is conducted via the platform (USURP [19]) created by the Bioengineering research group at the School of Engineering at the University of Leicester. The same platform is used to obtain HDF and phase analysis maps $[12,17]$.

\subsection{Combining Ionic Computer Models}

3 different Action Potential Ionic computer models were chosen:

- $\quad$ Courtemanche et al. 1998 [6];

- $\quad$ Fenton \& Karma 1998 [9];

- Simitev \& Biktashev (a variation of Courtemanche et allii's model) 2006 [13].

The development of the computer algorithm was completed by merging the algorithms of those three models into one. This was coded into a MATLAB script that can then be run on ECG \& AEG retrospective data. The final model is comprised from the amalgamation of the differential equations from each individual model.

\subsection{Statistical Analysis}

Statistical Analysis was performed using Windows software 'Graph pad Prism' (V7.04), to compare the data from generated HDF \& phase analysis values and values generated via Simulink.

\section{Results}

Using code combining the 3 models on MATLAB retrieved $3 \mathrm{D}$ simulations for all 10 patients at $79 \pm 5 \%$. Propagation route coincided with HDF regions in $66 \%$ of patient data. Phase analysis maps visually compared over simulations showed $57 \%$ of expected re-entry wavelets at points of heightened electrical activity.

\section{Discussion}

This study is a snapshot into the potential modelling techniques that can be developed to better tailor ablative strategies. One key limitation of AF guided ablation therapy has been the high level of subjectivity on determining which area to ablate. Which still persists even with using phase analysis and DF maps as validating factors. Thus, such work on modelling can create an additional verifying factors increasing the degree of objectivity.

More work needs to be completed to make the algorithm in the independent study more robust. For example, propagation tracking via Simulink only occurred around $80 \%$. Although this percentage seems high, the number of actual patients is only 10 . Thus, more work needs to be done to refine parameters and catch anomalies. Also, the results show that validating factors of comparisons to phase analysis maps and DF maps show mutually around $60 \%$ of electrical activity tracking which suggests that other electrophysiological anomalies may also contribute to electrical activity. It may be worth looking into fibrosis to explain this in future work.

A benefit of this study is that it used popular action potential (AP) computer models which can be easily applied, and their parameters changed. This can help in making the model more robust in future work. A drawback of this work is that the participant pool is small (only 10 patients). However, this will be addressed in future research because patient recruitment and trials have already begun for further studies.

A further criticism of this investigation is scalability. This study attempts to use ionic models at the cellular level for computer modelling simulations, to mathematically scale that to the whole of the left atrium specifically on trailing electrical activity is very difficult. Electrophysiological differences in each case highlight this. To address this further work should be completed in making the model more robust at the ionic level for a firm start to scale up towards a whole atrium model.

Another shortcoming of this investigation is the robustness of the Courtemanche et allii's model within the combined model in Simulink. Another ionic model (Nygren et al.) may be a better alternative and this can be tested in future work.

\section{Conclusion}

The study addresses some gaps in current research on AP modelling in AF, from lack thereof to execution. The study takes a novel approach by merging popular computer models into one algorithm and with the assistance of clinical data shows the importance of its 
further development to reduce subjectivity in ablation procedures, making the treatment of AF via catheter ablation more efficient. The approach has potential applications to other forms of AF therapy too.

\section{References}

1. Aronis, K., Ali, R. and Trayanova, N. (2019). The role of personalized atrial modeling in understanding atrial fibrillation mechanisms and improving treatment. International Journal of Cardiology, 287, pp.139-147.

2. Alonso, S., Bär, M. and Echebarria, B. (2016). Nonlinear physics of electrical wave propagation in the heart: a review. Reports on Progress in Physics, 79(9), p.096601.

3. American Heart Association (2019). Principal Concepts of AF Mechanisms. [image] Available at: https://www.ahajournals.org/doi/10.1161/circresaha.116.31 0489 [Accessed 4 May 2019].

4. Bhf.org.uk. (2019). Atrial fibrillation (AF) - Causes, Diagnosis, Symptoms \& Treatments. [online] Available at: https://www.bhf.org.uk/informationsupport/conditions/atria 1-fibrillation [Accessed 25 Jan. 2019].

5. Belmiloudi, A. and Corre, S. (2019). Mathematical modelling and analysis of dynamic effects of multiple time-varying delays on electrophysiological wave propagation in the heart. Nonlinear Analysis: Real World Applications, 47, pp.18-44.

6. Courtemanche, M., Ramirez, R. and Nattel, S. (1998). Ionic mechanisms underlying human atrial action potential properties: insights from a mathematical model. American Journal of Physiology-Heart and Circulatory Physiology, 275(1), pp.H301-H321.

7. De Bakker, J. and Wittkampf, F. (2010). The pathophysiologic basis of fractionated and complex electrograms and the impact of recording techniques on their detection and interpretation. Circulation: Arrhythmia and Electrophysiology, 3(2), pp.204-213.

8. Dharmaprani, D., McGavigan, A., Chapman, D., Kutlieh, R., Thanigaimani, S., Dykes, L., Kalman, J., Sanders, P., Pope, K., Kuklik, P. and Ganesan, A. (2019). Temporal stability and specificity of high bipolar electrogram entropy regions in sustained atrial fibrillation: Implications for mapping. Journal of Electrocardiology, 53, pp.18-27

9. Fenton, F. and Karma, A. (1998). Vortex dynamics in three-dimensional continuous myocardium with fiber rotation: Filament instability and fibrillation.

10. Grandi, E., Dobrev, D. and Heijman, J. (2019). Computational modeling: What does it tell us about atrial fibrillation therapy? International Journal of Cardiology, 287, pp.155-161
11. Peñaranda, A., Cantalapiedra, I., Bragard, J. and Echebarria, B. (2012). Cardiac dynamics: a simplified model for action potential propagation. Theoretical Biology and Medical Modelling, 9(1).

12. Salinet, J., Schlindwein, F., Stafford, P., Almeida, T., Li, X., Vanheusden, F., Guillem, M. and Ng, G. (2017). Propagation of meandering rotors surrounded by areas of high dominant frequency in persistent atrial fibrillation. Heart Rhythm, 14(9), pp.1269-1278.

13. Simitev, R. and Biktashev, V. (2006). Conditions for propagation and block of excitation in an asymptotic model of atrial tissue. Biophysical Journal, 90(7), pp.2258-2269.

14. Spach, M. and Kootsey, J. (1983). The nature of electrical propagation in cardiac muscle. American Journal of Physiology-Heart and Circulatory Physiology, 244(1), pp.H3-H22.

15. Tedrow, U. and Stevenson, W. (2011). Recording and interpreting unipolar electrograms to guide catheter ablation. Heart Rhythm, 8(5), pp.791-796.

16. Ugarte, J., Tobón, C. and Orozco-Duque, A. (2019). Entropy Mapping Approach for Functional Reentry Detection in Atrial Fibrillation: An In-Silico Study. Entropy, 21(2), p.194.

17. Almeida, T., Chu, G., Bell, M., Li, X., Salinet, J., Dastagir, N., Tuan, J., Stafford, P., André Ng, G. and Schlindwein, F. (2017). The temporal behavior and consistency of bipolar atrial electrograms in human persistent atrial fibrillation. Medical \& Biological Engineering \& Computing, 56(1), pp.71-83.

18. Almeida, T.P., Schlindwein, F.S., Salinet, J.L., Li, X., Chu, G., Tuan, J., Stafford, P.J., Ng, G.A. and Soriano, D. (2018). Characterization of human persistent atrial fibrillation electrograms using recurrence quantification analysis. Chaos: An Interdisciplinary Journal of Nonlinear Science, 28(8), p.085710.

19. Li, X., Salinet, J.L., Almeida, T.P., Vanheusden, F.J., Chu, G., Ng, G.A. and Schlindwein, F.S. (2017). An interactive platform to guide catheter ablation in human persistent atrial fibrillation using dominant frequency, organization and phase mapping. Computer Methods and Programs in Biomedicine, 141, pp.83-92.

Address for correspondence:

Saaima Ahmad.

University of Leicester

School of Engineering

University Rd, Leicester LE1 7RH.

sa841@le.ac.uk 